\title{
Design and Implementation of a TEM Stripline for EMC Testing
}

\author{
Sezgin Hilavin and Alp Kustepeli
}

\begin{abstract}
In this paper, a stripline for radiated field immunity testing of audio/video products is designed, constructed, calibrated, and verified. Since the canonical one is only suitable for testing equipments with height less than $70 \mathrm{~cm}$, there is a need of a new device which is also in compliance with EN 55020 standard for testing new large sets, like $47^{\prime \prime}$ thin-film transistor liquid crystal display (TFT LCD) television sets, in the frequency range of $150 \mathrm{kHz}-150 \mathrm{MHz}$. Increasing the height and width of testing area causes important problems in terms of field uniformity regarding the higher order modes, characteristic impedance, and reflections in addition to the room resonances and field interference sources like corners at the edges of stripline. Comprehensive numerical study is performed to overcome these problems and obtain the optimum design before the construction. Measured data show that the new stripline is in very good agreement with the specifications determined by the standards and therefore it can be used for the electromagnetic compatibility (EMC) testing.
\end{abstract}

Index Terms-Electromagnetic compatibility (EMC), immunity, susceptibility, stripline, EN 55020, EN 61000-4-20.

\section{INTRODUCTION}

$\mathbf{E}$ $\mathrm{ACH}$ electrical product must have an adequate level of immunity to the electromagnetic disturbances in its environment. Even though different tests can be employed for validation, they must be conducted according to some specific standards to be in compliance with the electromagnetic compatibility (EMC) directive. Ideally, every product should be tested in planar electric fields which are uniform in both phase and amplitude. If the field is not uniform enough in the frequency range of operation, the test results highly deviate from test to test.

The stripline method is one of the most important procedures used in EMC tests. Roseberry and Schulz investigated its use in radio frequency (RF) susceptibility tests [1]. As shown in Fig. 1, a stripline consists of two parallel conducting plates in which the equipment under test (EUT) is placed. They showed that it generates uniform fields over RF spectrum and allows one to know the magnitude of test field more accurately than if an antenna

Manuscript received April 1, 2013; accepted June 4, 2013. Date of publication July 26, 2013; date of current version January 27, 2014. This work was supported by Vestel Electronics.

S. Hilavin was with the Department of Electrical and Electronics Engineering, Izmir Institute of Technology, Izmir 35430, Turkey. He is now with Vestel Electronics, Manisa 45030, Turkey (e-mail: sezgin.hilavin@ vestel.com.tr).

A. Kustepeli is with the Department of Electrical and Electronics Engineering, Izmir Institute of Technology, Izmir 35430, Turkey (e-mail: alpkustepeli@ iyte.edu.tr).

Digital Object Identifier 10.1109/TEMC.2013.2271834

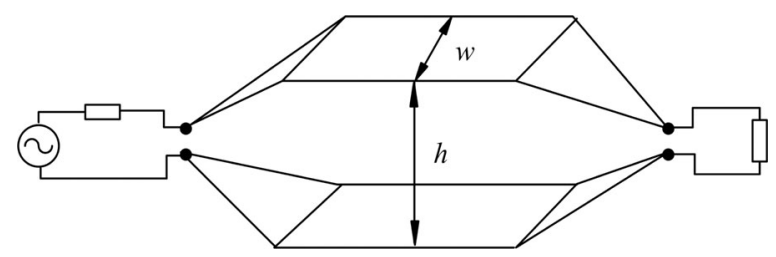

Fig. 1. Basic configuration for an open stripline with source and terminating impedance.

were used as a field source in an anechoic chamber. Moreover, stripline requires less power when compared with the chamber because the electric field is concentrated mostly between the plates and it can be placed in a simple shielded room, which means there is no need for an expensive anechoic chamber. The high precision and repeatability of tests are the other advantages of stripline method. Actually, a stripline can be considered as an open or nonshielded version of a transverse electromagnetic (TEM) cell, which was first described by Crawford in 1974 [2]. TEM cells are frequently used for immunity measurements and there are even some specific studies to determine the emission levels of integrated circuits by using them [3], [4]. Since stripline is a special type of TEM cell, it is exactly used in the same manner and has most of the same limitations [5]-[9]. The field in a stripline can be disturbed because of the higher order modes, reflections, and room resonances. Even though they cannot be eliminated completely, suppression to an accaptable degree can be achieved by placing absorbing materials in and around the stripline [10]. The stripline method is preferred and widely used in many industrial areas for EMC tests because of its advantages when compared with anechoic chambers.

The canonical stripline recommended by EN 55020 [11] has $80 \mathrm{~cm}$ space between parallel conductors allowing for the test of equipments with height less than $70 \mathrm{~cm}$ which is not enough to test new large products. For example, a 47" thin-film transistor liquid crystal display television (TFT LCD TV) set is about $1 \mathrm{~m}$ high and cannot be tested in available commercial striplines. Obtaining a stripline with a large usable area is not possible by just scaling the EN 55020 stripline. The problem is that the field uniformity cannot be created in the testing volume especially due to the first transverse electric (TE) mode in the test frequency range. It is acceptable to use another TEM device according to EN 55020 if the test results obtained from the two do not differ by more than $2 \mathrm{~dB}$ for the same EUT. Therefore, in this study, it is intended to design, construct, calibrate, and verify a new stripline which is in compliance with the required specifications and suitable for the EMC tests of large products. 


\section{DESIGN AND CONSTRUCTION}

In a stripline, in addition to the TEM mode, TE and transverse magnetic (TM) modes also propagate with the cutoff frequencies roughly determined by the cross section of stripline. One of the effects disturbing the field uniformity in stripline is the existence of higher order modes, especially the first TE mode, and these undesired modes become an important problem when they are supported by the line if any one of the cross-sectional dimensions exceeds a half wavelength [12]. For this reason, it is aimed to generate only TEM mode through the line in the application of the stripline method. The cutoff frequency of the first TE mode, the most effective and dominant mode in terms of field uniformity because the cutoff frequencies of the others are much higher, is approximately given by [13]

$$
f_{c}=\frac{300}{\sqrt{\varepsilon_{r}}(2 w+0.8 h)}
$$

where $w$ is the width of the upper conductor, $h$ is the distance between the upper and lower conductors, and $\varepsilon_{r}$ is the relative dielectric constant of the substrate used between the conductors. In (1), $w$ and $h$ are both in meters and $f_{c}$ is in megahertz. If the equation is examined, one can see that the certain frequency range of operation is primarily determined by the dimensions, and they are chosen so that the first cutoff frequency is above the maximum test frequency. Since $w=0.6 \mathrm{~m}$ and $h=0.8 \mathrm{~m}$ for the standard stripline recommended by EN 55020, its first cutoff frequency is obtained as $163 \mathrm{MHz}$ which is out of the test frequency range. But if the height is increased, the cutoff frequencies of the modes are decreased, particularly that of the first and most important one has to be in the considered range. The characteristic impedance of stripline, $Z_{0}$, is also determined by the dimensions and it is given as [14]

$$
Z_{0}=\frac{60}{\sqrt{\varepsilon_{e}}} \ln \left(\frac{8 h}{w}+\frac{w}{4 h}\right) \text { for } w / h \leq 1
$$

where $\varepsilon_{e}$ is the effective dielectric constant which is given by

$$
\varepsilon_{e}=\frac{\varepsilon_{r}+1}{2}+\frac{\varepsilon_{r}-1}{2 \sqrt{1+12 h / w}} .
$$

Since the stripline is placed in air, $\varepsilon_{r}=1$, and $\varepsilon_{e}$ is obtained as 1 by using (3). In this case, if the stripline is designed as its characteristic impedance to be $50 \Omega$, it results in a narrow width or low height and it would not provide necessary testing volume [5]. Therefore, even for the standard stripline, $Z_{0}$ cannot be chosen as $50 \Omega$. For the dimensions used for the standard stripline, the characteristic impedance of the line is obtained as $143 \Omega$. However, the internal impedance of the signal source is $50 \Omega$, and a matching network is used to decrease the mismatch between the source and the line [11]. The characteristic impedance determines the voltage across the plates for a given input power and hence the electric field intensity inside the stripline, which is obtained by the ratio of the plate voltage to the plate separation [15]. EN 55020 describes the setup, shown in Fig. 2, to establish a specific electric field at the center of the test volume. The matching network at the feed point and the termination at the end of the line reduces the mismatch level [12], [15]. The induced voltage at the measuring plate is measured with an RF

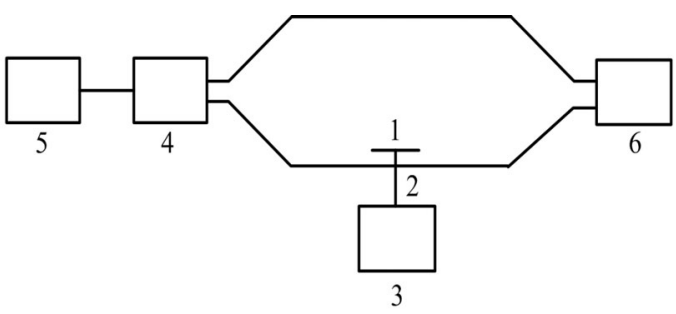
1. Measuring plate $(200 \pm 0.5) \mathrm{mm} \times 1 \mathrm{~mm}$
2. Measuring probe
4. Matching network
3. RF millivoltmeter
5. Signal generator
6. Termination resistor $150 \Omega$

Fig. 2. Circuit arrangement for the calibration of the system.

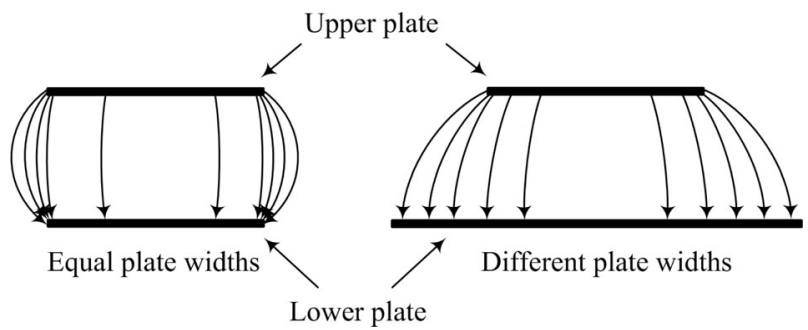

Fig. 3. Fringing fields at the edges of a two-plate open stripline for two different applications.

millivoltmeter and the field intensity in the stripline is $3 \mathrm{~V} / \mathrm{m}$ if the measured plate voltage is within the limits given by the standard. The test is performed for the whole test frequency range and deviations more than $\pm 2 \mathrm{~dB}$ in the calibration curve are taken into account by the correction factor

$$
K_{1}=\frac{U_{m}}{U_{n}}
$$

where $U_{m}$ is the measured voltage value at the measuring plate and $U_{n}$ is the nominal voltage value.

The widths of plates are also important for the field uniformity. Two plate striplines can have equal plate widths or have the lower one wider than the top one as illustrated in Fig. 3. If the widths are equal, there are bowings at the edges of the plates and this prevents the field concentration inside the stripline. If the lower plate is wider than the upper one, the fringing fields are more perpendicular to the lower one and they increase the uniformity [12]. This special feature which is used in the standard stripline is also employed in the design of new stripline. The uniform area is described by the standard EN 61000-4-20 [16] and it is the volume between the two virtual vertical planes, which are orthogonal to the propagation direction of the field, as shown with the points in Fig. 4. The planes are $0.5 \mathrm{~m}$ away from the center of the stripline. This length was suitable to keep the TV in usable test volume which is the uniform area between these planes. Six points with $0.5 \mathrm{~m}$ distance between them were selected on both planes as shown in the figure. Two points were in horizontal and three points are in vertical orientation. The evaluation of the uniformity is made by checking the power required to create the same field level at each point. Then, the maximum power difference between the points in each plane is 


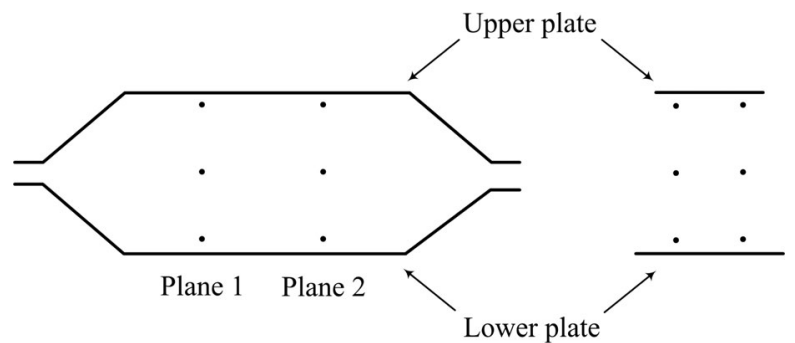

(a)

(b)

Fig. 4. Measurement points in plane 1 and in plane 2 for the uniformity test. (a) Side view. (b) Cross-sectional view.

found for the whole test frequency range. According to the $6 \mathrm{~dB}$ criterion, maximum difference at each frequency must be less than $6 \mathrm{~dB}$ for at least $75 \%$ points. Since there are six points in each plane, maximum difference of five points must be less than $6 \mathrm{~dB}$ for each plane.

There are also other important problems caused by the termination, tapered sections and feed point. A $150 \Omega$ termination resistor is used to decrease the reflections at the end of the standard stripline. Due to the tapered sections, the wave launched at the feed exhibits a spherical wavefront and results in considerable variation in phase across the width of the line, particularly at the transition between the tapered section and the beginning of parallel plate region, and field interference can also be generated at the edges of these transitions [12]. In addition to these, there are resonances caused by the stripline itself or the room in which it is placed. To obtain a uniform field even inside the standard stripline, they are suppressed by using absorbing material in and around the stripline. All of the problems mentioned previously are determinant on the operating frequency range and the field uniformity; therefore, they must be taken into consideration and overcome in the design of stripline carefully.

According to the information given previously, one can conclude that the dimensions $w$ and $h$ of stripline are two parameters to be adjusted and optimized in accordance with design needs. In order to test large TFT LCD products up to $47^{\prime \prime}$ which are about $1 \mathrm{~m}$ high, the plates of the stripline should be at least $1.2 \mathrm{~m}$ apart for a suitable usable area. For this reason, the height of the stripline $h$ was chosen as $1.2 \mathrm{~m}$. The reason for not increasing the height, more than a suitable one, is that more power is needed to obtain the same electric field intensity in the line and a smaller width is obtained for the same cutoff frequency. If one calculates the width of the line such that the cutoff frequency of the first mode to be $150 \mathrm{MHz}$ with the height specified, it is found that $w=0.52 \mathrm{~m}$ which is even smaller than the width of the standard line and is not suitable for the tests of large products. Furthermore, the characteristic impedance of the line is obtained as $175 \Omega$ which increases the mismatch at both ends of line because special RF power resistors are used for the termination, which is $150 \Omega$, and for the matching network, which matches 150 to $50 \Omega$, in order to get sufficient flat RF responses in the testing frequency range. If the width of the standard line, $w=0.6 \mathrm{~m}$, is used for the new one, the cutoff frequency of the first mode is obtained as $139 \mathrm{MHz}$ from (1).

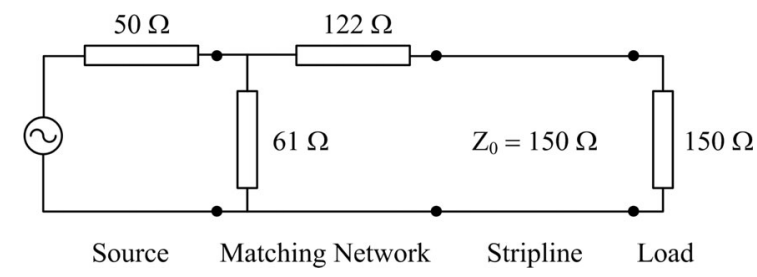

Fig. 5. Block diagram of the stripline testing system.

It seems that it might be suitable in terms of obtaining a TEM field since that value is very close to the end of the frequency range of operation but one must also consider the characteristic impedance which is obtained as $167 \Omega$. Even though it is seen that all of these dimensions and values are not appropriate for the optimum design, they were taken into consideration at the beginning of the design process. Simulations were performed by using EMCOS software package [17]. It was seen in the preliminary numerical study that it was better to decrease the reflections at the end of the line. Therefore, it was concluded and decided to choose the characteristic impedance of the line as to be $150 \Omega$. Accordingly the width is obtained about $0.8 \mathrm{~m}$ and the cutoff frequency of the larger stripline is about $117 \mathrm{MHz}$ with the new dimensions, $w=0.8 \mathrm{~m}$ and $h=1.2 \mathrm{~m}$. This means that TE and TM waves exist after $117 \mathrm{MHz}$. In fact, there is no possible solution to remove the modes from the testing frequency range completely. However, their effects on the field uniformity could be slightly suppressed. Reducing their impact on the field uniformity by using some absorbers is a helpful solution [18] If enough attenuation can be obtained inside the stripline, then the field uniformity conditions accepted by the standard could be fulfilled.

The characteristic impedance of the line is different from the source impedance and a matching network is used at the source side. The matching network and circuit equivalent of the whole system are given in Fig. 5. The widths of upper and lower plates are also chosen differently and the lower plate's width was determined as $1.1 \mathrm{~m}$ from the simulations. In view of the fact that the discontinuity parts disturb the field uniformity, the tapered parts must be far from the testing volume and the length of the line is taken as $2.4 \mathrm{~m}$. After the dimensions and characteristic impedance were determined, simulations were again performed to improve the performance of the stripline by making the other adjustments before realizing it. The design was obviously examined according to EN 55020, EN 61000-4-20, and voltage standing wave ratio (VSWR) by using the configurations given in Figs. 2 and 4. All of the simulations were performed exactly in the same manner as to be done in the measurements. In order to find the field strength in the test volume, a field probe was inserted and the plate voltage was computed via a voltage probe in the simulation. It was seen that there were some resonances around 100-120 MHz as expected and the extension of useful frequency range was achieved by absorber loading. Many different dimensions and placements of the absorbers were applied in the simulations to optimize the configuration by considering their electrical properties [19]. The simulations showed that the dielectric properties of the absorbers highly affected the results 


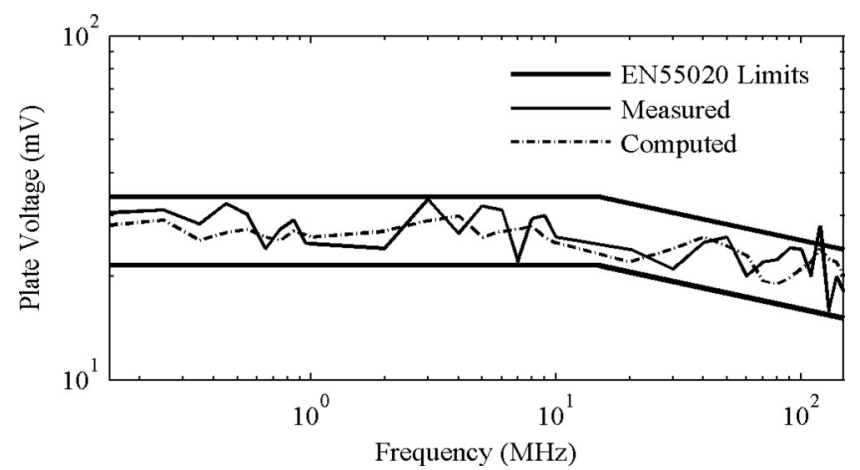

Fig. 6. Plate voltage for the stripline.

as well as their shapes and locations. Based on the simulation trials, $20 \mathrm{~cm}$ pyramidal absorbers were used, and they were placed around the open sides and at the tapered sections. However, resonances still existed about $110 \mathrm{MHz}$ similar to the simulation results. This problem was solved by using hybrid absorbers which are made of ferrite tiles and RF pyramidal absorbers together to strengthen the attenuation performance. They improve the uniformity of the field inside by increasing the vertical component of the electrical field at the edges of the line [20]. Finally, the stripline was constructed with the dimensions and the special arrangements of the absorbers according to the results obtained from the detailed numerical study.

\section{CALibRation AND Verification}

After the construction, the calibration of the new stripline was made as specified by the standard EN 55020. An electric field probe was placed at the centre of the line. The input voltage was changed until $3 \mathrm{~V} / \mathrm{m}$ electrical field strength was obtained at $15 \mathrm{MHz}$. By using an RF amplifier the power was kept constant in the whole test frequency range and an RF millivoltmeter was placed in the center of the measuring plate to get the calibration voltage. The calibration curve is given in Fig. 6 together with the limits of the standard. The computed results are also presented in the figure. Even though the measured plate voltage is out of the limits for only a few frequencies at the very end of the test frequency range, the correction factor $K_{1}$ is used at these frequencies for the tests of products. The measured field uniformity test results for both planes are presented in Fig. 7. One can see that the power difference at each frequency is in the $6 \mathrm{~dB}$ tolerance range for both 5 points and 6 points. Therefore, the criterion is actually satisfied for $100 \%$ of the points in the test planes, which is a very satisfactory result in terms of the field uniformity.

The measured VSWR of the new stripline shown in Fig. 8 was obtained by using a Rohde \& Schwarz ZVL network analyzer. The worst value of the VSWR is 1.23 at $150 \mathrm{MHz}$, at the very end of the test range. From the figure, it is seen that the matching performance of the whole system is also very satisfactory. The efficiency is better than a usual test system in an anechoic chamber in which a broadband antenna is used and has high standing wave ratios. This was also approved by measuring the forward and the reversed power during the field uniformity testing.

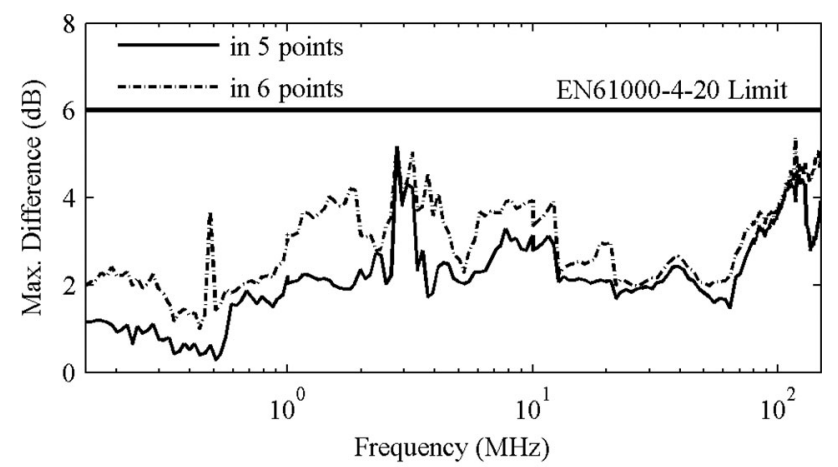

(a)

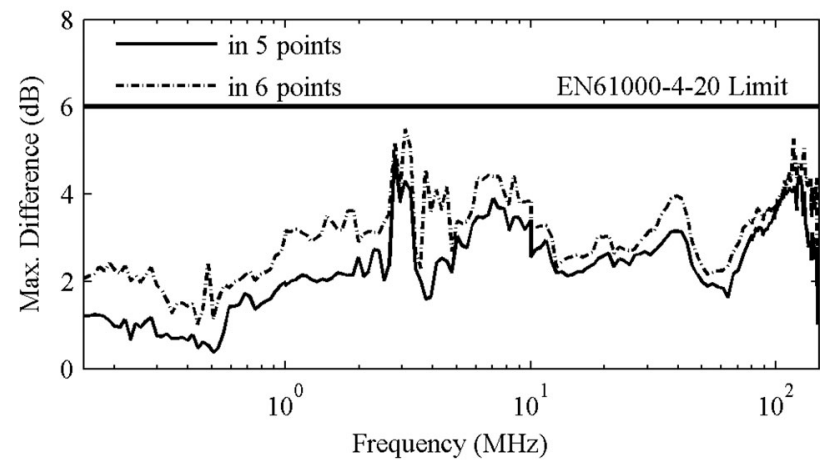

(b)

Fig. 7. Measured maximum power differences (a) in plane 1 and (b) in plane 2 for the field uniformity test.

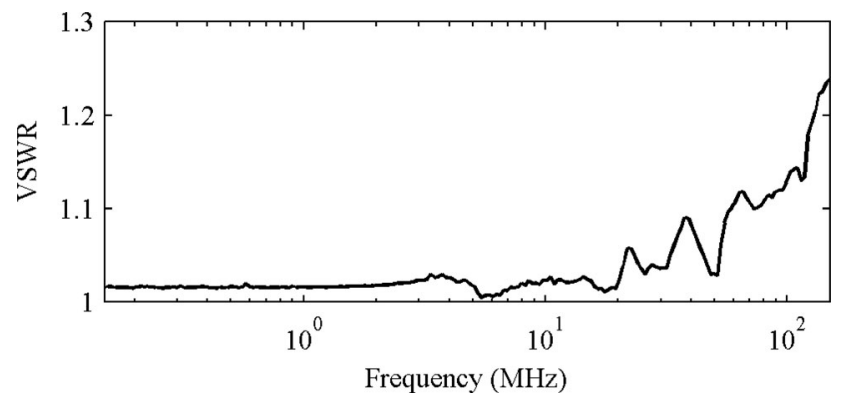

Fig. 8. Measured VSWR of the system.

Finally for the comparisons and verification purposes of the new stripline, the difference between the equivalent tests of the same EUT was examined by using a TV set and it must be below $2 \mathrm{~dB}$ as specified by EN 55020. The same observations occurred at the same frequency for the EUT which was tested in both the standard and the new stripline. The difference of the disturbance levels between two tests was about $1 \mathrm{~dB}$. Therefore, it can be concluded that this new stripline can be used for the testing of wide range of products which require high testing volume and high field uniformity.

\section{CONCLUSION}

In this study, a new TEM stripline was designed for the testing of immunity to radiated fields of large EUTs, which cannot be tested in a standard stripline, in the frequency range of 
$150 \mathrm{KHz}-150 \mathrm{MHz}$. A simulation program was used for the detailed numerical study to optimize the stripline in terms of the standards, EN 55020 and EN 61000-4-20, and the VSWR before the realization. After the construction, it was seen that the performance of the new stripline was very satisfactory and the correction factor $K_{1}$ needed to be used at only a few frequencies. The most important and challenging part of the design was to obtain the field uniformity in the stripline because it was not possible to move the first TE mode outside of the usable frequency range due to the large dimensions. Its disturbing effect was suppressed by using absorbers with special placements. The verifications and comparisons were also made according to the criterion specified by the standard. From the measurement results it is seen that the new TEM stripline is in compliance with requirements specified by the standards, especially in terms of the field uniformity, and therefore it can be used as a compatible test site for immunity to radiated fields.

\section{ACKNOWLEDGMENT}

The authors would like to thank Vestel Electronics for the usage of their R\&D laboratory facilities during this research.

\section{REFERENCES}

[1] B. E. Roseberry and R. B. Schulz, "A parallel-stripline for testing of RF susceptibility," IEEE Trans. Electromagn. Compat., vol. 7, no. 2, pp. 142 150, Jun. 1965.

[2] M. L. Crawford, "Generation of standard EM fields using TEM transmission cells," IEEE Trans. Electromagn. Compat., vol. EMC-16, no. 4, pp. 189-195, Nov. 1974.

[3] S. Deng, T. Hubing, and D. G. Beetner, "Characterizing the electric field coupling from IC heatsink structures to external cables using TEM cell measurements," IEEE Trans. Electromagn. Compat., vol. 49, no. 4, pp. 785-791, Nov. 2007.

[4] F. Fiori and F. Musolini, "Measurement of integrated circuit conducted emissions by using a transverse electromagnetic mode (TEM) cell," IEEE Trans. Electromagn. Compat., vol. 43, no. 4, pp. 622-628, Nov. 2001.

[5] Y. C. Chung, T. W. Kang, and D. C. Park, "Design and construction of stripline for measuring electromagnetic immunity of vehicular electrical cables," in Proc. Electromagn. Compat. Int. Symp., 1997, pp. 9-12, paper: 10.1109/ELMAGC.1997.617042, 1997.
[6] Z. Jingjun and F. Junmei, "Higher order mode cutoff frequencies in TEM cells calculated with TLM method," IEEE Trans. Electromagn. Compat., vol. 30, no. 4, p. 563-566, Nov. 1988.

[7] P. F. Wilson and M. T. Ma, "Simple approximate expressions for higher order mode cutoff and resonant frequencies in TEM cells," IEEE Trans. Electromagn. Compat., vol. 28, no. 3, pp. 125-130, Aug. 1986.

[8] J. Kuveda and R. K. Libla, "Determination of frequency range limit when using stripline as emission or immunity test methods," in Proc. IEEE Int Symp. Electromagn. Compat., Aug. 2006, vol. 3, pp. 823-828.

[9] R. Lampe, P. Klock, D. Tanner, and P. Mayes, "Analysis and experiment concerning the cutoff frequencies of rectangular striplines," IEEE Trans. Microw. Theory Tech., vol. MTT-34, no. 8, pp. 898-899, Aug. 1986.

[10] M. Valek, T. Korinek, and T. Bostik, "Design of stripline for EMC testing," in Proc. 14th IEEE Conf. Microw. Tech., 2008, pp. 1-4.

[11] Sound and Television Broadcast Receivers and Associated EquipmentImmunity Characteristics-Limits and Methods of Measurement Specification, CENELEC Standard EN 55020, 2007.

[12] ETSI Technical Committee Electromagnetic compatibility and Radio spectrum Matters, "Improvement on radiated methods of measurement (using test site) and evaluation of the corresponding measurement uncertainties; part 5: striplines," ETSI, France, Tech. Rep. TR 102 273-5 V1.2.1, 2001-12.

[13] M. Golio and B. Raton, The RF and Microwave Handbook. Baco Raton, FL, USA: CRC Press, 2001.

[14] D. M. Pozar, Microwave Engineering, 2nd ed. New York, NY, USA: Wiley, 1998.

[15] D. Morgan, A Handbook for EMC Testing and Measurement, 1st ed. Milton Keynes, U. K.: The Institution of Engineering and Technology, 1994.

[16] Electromagnetic Compatibility (Emc) - Part 4-20: Testing and Measurement Techniques - Emission and Immunity Testing in Transverse Electromagnetic (Tem) Waveguides, CENELEC Standard EN 61000-4-20, 2010

[17] EMC studio electromagnetic simulation software, ver.6.0.1, EMCOS, Tbilisi, Georgia, 2010.

[18] W. Bittinger, "Properties of open strip lines for EMC measurements," in Proc. IEEE Int. Symp. Electromagn. Compat., Dallas, TX, USA, Aug. 9-13, 1993, pp. 120-125.

[19] K. Shimada, T. Hayashi, and M. Tokuda, "Fully compact anechoic chamber using the pyramidal ferrite absorber for immunity test," in Proc. IEEE Int. Symp. Electromagn. Compat., 2000, vol. 1, pp. 225-230.

[20] K. Malaric and J. Bartolic, "Design of a TEM-cell with increased usable test area," Turkish J. Elect. Eng. Comput. Sci., vol. 11, no. 3, pp. 143-154, 2003.

Authors' photographs and biographies not available at the time of publication. 